\title{
A STUDY ON THE SAFETY AND EFFICACY OF MONOGLYCERIDE OF LAURIC ACID AS ADJUVANT TREATMENT IN PERSONS LIVING WITH HIV INFECTION
}

\author{
By \\ Eric A. Tayag ${ }^{1}$, Edna G. Santiago ${ }^{1}$, Minda A. Manado ${ }^{l}$ Perla N. Alban ${ }^{l,}$ Dorothy \\ Mae Agdamag ${ }^{1,}$ Suzette Lazo ${ }^{1}$, Amelito Adel, ${ }^{1}$ Rosario Tactacan, ${ }^{1}$ Alicia O. \\ Caspellan, ${ }^{1}$, Conrado Dayrit ${ }^{2}$, Noel Espallardo ${ }^{2}$
}

\section{INTRODUCTION}

The AIDS pandemic has caused global concern what with its threat to man's survival and the enormous cost to prevent and treat the illness. No effective cure is possible but for the last fifteen years, countless studies were made to improve survival, delay disease progression or just improve the quality of life. Various clinical trials were designed to inhibit specific processes that are necessary for the human immunodeficiency virus (HIV) to survive the internal milieu. As important as these discoveries, are the precise methods of measuring the impact of these treatments. Only in the last five years has there been a better understanding of these processes and methods.

Currently, the most acceptable treatment regimen to delay progression to AIDS constitutes combination therapy to which fewer individuals have access to, especially those in developing countries. In the Philippines, treatment is focused in the prevention and treatment of opportunistic infections, which require periodic evaluation and sometimes prolonged confinements in hospitals. At San Lazaro Hospital (SLH), at least three clinical trials were done to study the effects of these drugs. To date none has been recommended because government cannot afford life-long treatment of persons living with HIV infection. Thus, the use of these combinations is limited to patients who are enrolled in these studies and only for a limited period of time. Concerns about some serious side-effects relative to their use have also made these treatments unpopular.

Recently, alternative therapies have been evaluated although none have become acceptable. They are now available as herbs or plant extracts. Interest has not waned regarding their use because safety and cost have become more important than the clinical benefits. When news regarding the benefits of coconut was reported by persons living with HIV infection from the Caribbean, it was just a matter of time before researchers would throw their attention to this tropical plant.

The human immunodeficiency virus (HIV) has a lipid envelope vulnerable to attack by fatty acids and monoglycerides, which act by altering cell permeability. Lauric acid lipid or monolaurin, a medium-chain monoglyceride, has been shown to possibly possess this antiviral property which is quite different from what current available antiretroviral drugs are capable of doing; attacking the viral genome. Monolaurin is found in coconut oil. Presently, monolaurin is commercially available in high purity (>90\%) and because it is nutrient, it is virtually non-toxic. A study among Filipino healthy persons was done to assure its tolerability and safety prior to this study.

This initial study is aimed at establishing the safety and efficacy of the monoglyceride of lauric acid as adjuvant treatment in persons living with HIV infection. Both coconut oil and monolaurin were used because we wanted to be certain that it is monolaurin, which possesses the anti-retroviral properties.

1 San Lazaro Hospital, Manila, Philippines.

2 United Laboratories, Manila, Philippines. 


\section{METHODS}

Fifteen adult patients (18 years and above) were each randomly assigned to one of three treatment groups that consisted of the following: Group I - coconut oil; Group II - low dose monolaurin and Group 3- high dose monolaurin. Coconut oil was given at $15 \mathrm{ml}$ three times daily while low dose monolaurin as $800 \mathrm{mg} /$ capsule, one capsule three times daily and high dose monolaurin as $800 \mathrm{mg} /$ capsules, three times daily. The duration of treatment was 6 months. Multivitamins (containing anti-oxidants) wee also given to all patients. The following were excluded from the study: pregnant or lactating mother; patients with CD4 count $<200$ cells $/ \mathrm{ml}$; those with severe renal or liver impairment; and, those with previous intake of anti-retroviral drug within two months of start of trial. An informed consent was required prior to enrolment into the study.

All patients were observed for clinical (clinical status, weight loss, progression to minor opportunistic infections, progression to AIDS and toxicity) and laboratory parameters (CBC, cholesterol panel, liver enzymes, BUN, creatinine, CD4 cell count and viral load). Laboratory tests were done at the San Lazaro Hospital STD/AIDS Central Cooperative Laboratory (SACCL). Repeated measurements were made during treatment (3 months after start of treatment) and at end of study (6 months after). A standard proforma was used to document progress of patient. Patients were regularly examined every month and were seen time to time when necessary since patients were instructed to report any observable clinical change. Most patients stayed at the Bahay Lingap (half way home for patients living with HIV infection) for the duration of the study. Treatment success was defined as reduced viral load and increased CD4 count at end of study with no or minor side reactions. Analysis was done using EPI-INFO version $6 \mathrm{~b}$ software.

\section{RESULTS}

Fifteen patients were recruited in the study. Five were males and age ranged from 22 years - 38 years. Baseline characteristics were basically similar (see Table 1). Viral load decreased in 6/15 (60\%) after 3 months of treatment and in 10/15 (66.7\%) at end of treatment (EOT) [see Tables 2 and 3]. The total log-change in viral load was increased in all groups 3 months after treatment and was reduced in all groups at EOT (see Table 6). Greatest reduction in a mean viral load at EOT was observed among those who took low dose monolaurin (Figure 1). CD4 count increased only in 3/15 $(20 \%)$ after 3 months of treatment and increased in $7 / 15(46.7 \%)$ at EOT (see Table 4). The total CD4 count decreased in all groups after 3 months of treatment (see Table 7). It increased only in the oil and high dose monolaurin groups at EOT. The mean CD4 count initially decreased during treatment but started to rise up to EOT in all treatment groups (see Figure 2).

Mean cholesterol levels increased but were within normal range in all groups at EOT (see Table 8). Mean triglyceride levels increased beyond normal levels in all groups at EOT. Mean HDL level increased only in the high dose monolaurin group at EOT and the increase was beyond normal levels. Mean LDL levels remained within normal levels in all groups at EOT but increase was noted in high dose monolaurin group. One patient in the high dose monolaurin group had sustained ALT and AST elevations. BUN, creatinine and hemoglobin did not significantly change in all groups at EOT.

Three patients progressed to AIDS (CD4 count $<200)$ after 3 months of treatment; two from the low dose of monolaurin group and one from group 1 (oil). One patient from the low dose monolaurin recovered (CD4 count $>200$ ) at EOT but the others did not. The patient, who took oil, temporarily stopped for 7 weeks because she developed severe ascites probably secondary to acute hepatitis. She died one month after EOT. Also, a minor opportunistic infection, oral thrush, was noted in another patient who received treatment with oil. There was generally weight gain. One patient from the low dose monolaurin group gained $23.5 \mathrm{~kg}$ at EOT. 
Adverse reactions were minimal and generally benign (see Table 10). Green urine was reported in patients who took the low dose and high dose monolaurin (available as green-colored capsules). The discoloration was intermittent and disappeared after 3 months. All groups reported flaring up of acne even at EOT. Ascites was reported in one patient who took oil. This was not related to treatment.

\section{DISCUSSION}

Treatment success using conventional combination anti-retroviral drugs now requires an observable decrease in viral load 2 months after treatment and a 2-log decrease, with no detectable virus, 6 months after treatment. CD4 counts were expected to recover or rise after the treatment period. Earlier in major clinical trials of anti-retroviral therapy, minimal rise in CD4 counts of 50 or more were already indicative of beneficial treatment.

The study demonstrated a reduced mean log-change in viral load of $0.13-0.40$; the reduction being greatest in the low dose monolaurin group. The mean log-change was low or slows in the "oil" group possibly because baseline levels were already low to begin with. Reduction in viral load correlates with probable anti-viral effects of coconut oil or monolaurin. The required 2-log reduction in viral load as a marker for treatment success did not occur because of the small sample size or that it may not be applicable to monolaurin. Extending treatment beyond six months may have produced better results. Or else, combining monolaurin with another anti-retroviral might produce the same results. In the case of existing anti-retroviral combinations, sustained reduction is anticipated over time as long as patients continue to carry on treatment beyond 6 months and resistance does not occur. The study is limited though to 6 months of treatment, the time required to recognize any benefit at all if there is any.

The total and mean CD4 counts declined initially in all treatment groups but an increase was noted at EOT. It is possible that CD4 will take more time to show any favorable changes. Perhaps, we must allow longer periods ( $>6$ months) to see these changes. However, two patients in the low dose monolaurin and one from the "oil" group progressed to AIDS (CD4 count $<200$ ). The patient who took oil then died one month after EOT, had an initial CD4 count $>200$. It was possible that shortly after treatment her CD4 count went below 200 and this was detected only after 3 months when the next test was performed. She was initially diagnosed 12 years ago before this trial. As for the rest, we cannot find a suitable explanation for their progression to AIDS since both were diagnosed just 5 years ago. Progression to AIDS takes about 8-10 years from time of diagnosis. Besides, the reduced viral load contradicts any understanding that treatment worsened the illness.

There is concern regarding the increased mean triglyceride levels despite the fact the patients were given anti-oxidants. Although there were no clear, attributable clinical abnormalities to these increased levels, we were also unable to fully explain why this happened. Our previous study with normal individuals did not show this finding. If this was a transient increase and was related to treatment, we expect levels to normalize once treatment is completed.

The human immunodeficiency virus (HIV) has a lipid envelope vulnerable to attack by fatty acids and monoglycerides, which act by altering cell permeability. Lauric acid lipid or monolaurin, a medium-chain monoglyceride, has been shown to possibly possess this antiviral property which is quite different from what current available antiretroviral drugs are capable of doing: attacking the viral genome. Monolaurin is found in coconut oil. Presently, monolaurin is commercially available in high purity $(>90 \%)$ and because it is a nutrient, it is virtually non-toxic.

San Lazaro Hospital, in collaboration with United Laboratories (UNILAB), and the Philippine Coconut Research and Development Foundation, Inc. (PCRDF), conducted a 6 monthclinical trial to evaluate the clinical safety and efficacy of monolaurin in persons living with HIV infection. 
Fifteen patients were recruited. Ages ranged from 22-38 years old; five were males. Eleven out of $15(73 \%)$ had reduced viral load, while 7/15 (46\%) has increased CD4 count. Twelve out of 15 had weight gain, which ranged from $1 \mathrm{~kg}-23 \mathrm{kgs} 6$ months after treatment. Among the treatment groups, decrease in viral load was observed in $4 / 5(80 \%)$ coconut oil, $4 / 5(80 \%)$ low dose monolaurin and $3 / 5(60 \%)$ high dose monolaurin. Increase in CD4 count was shown in $2 / 5(40 \%)$ coconut oil, 4/5 (80\%) low dose monolaurin and 2/5 (40\%) high dose monolaurin. Minor side effects were noted like self-limited skin rashes and furuncles.

Our preliminary analysis strongly suggests the possible antiretroviral property of coconut oil or monolaurin in persons living with HIV infection. This finding is encouraging because monolaurin is safe and widely available. The next step is to conduct a randomized controlled trial with more patients.

\section{CONCLUSION}

The study was able to show mean reductions in viral load with variable changes in CD4 counts in all treatment groups. Although this was not the desired minimum outcome (2-log decrease in viral load), we were able to demonstrate the potential anti-retroviral effects of coconut oil and monolaurin. Another study involving a bigger sample size should be undertaken if recommendations to its future use as adjuvant treatment for persons with HIV infection are to be made. And because monolaurin is contained in coconut oil future studies should therefore use only monolaurin.

\section{REFERENCES}

BARTLETT, J.G. The John Hopkins Hospital 1997 Guide to Medical Care of Patients with HIV Infection

MANDELL, G.L., BENNETT, J.E., DOLIN, R. Principles and Practice of Infectious Diseases, 1995: 1164-1305

SPOONER, K.M., LANE, H.C., MASUR, H. Guide to Major Clinical Trials of Antiretroviral Therapy Administered to Patients with Human Immunodeficency Virus. Clinical Infectious Diseases. 1996; 23: 15-27

KABARA, J.J. et al. Unsaturation in Fatty Acids as a Factor for Antimicrobial Action. J. Med. Chem. 1972. 16:1

KOHN, A., GITELMAN, J. INBAR, M. Interaction of Polyunsaturated Fatty Acids With Animal Cells And Enveloped Viruses. Antimicrobial Agents Chemother. 1980; 189: 962-968

HORNUNG B., AMTMANN, E., SAUER, G. Lauric Acid Inhibits the Maturation of Vesicular Stomatitis Virus. Virology 1994; 75: 353-361. 
Table 1. Baseline characteristics of 3 treatment groups. Monolaurin clinical trail

\begin{tabular}{|c|c|c|c|}
\hline VARIABLES & Coconut Oil & $\begin{array}{c}\text { Low Dose } \\
\text { Monolaurin }\end{array}$ & $\begin{array}{l}\text { High Dose } \\
\text { Monolaurin }\end{array}$ \\
\hline $\begin{array}{c}\text { Gender distribution } \\
\text { Male (no/\%) } \\
\text { Female (no/\%) }\end{array}$ & $\begin{array}{l}1(20) \\
4(80)\end{array}$ & $\begin{array}{l}2(40) \\
3(60)\end{array}$ & $\begin{array}{l}2(40) \\
3(60)\end{array}$ \\
\hline $\begin{array}{l}\text { Weight (kgs) } \\
\text { Mean } \\
\text { Range }\end{array}$ & $\begin{array}{r}49.2 \\
39.0-58.0\end{array}$ & $\begin{array}{r}56.5 \\
49.0-68.0\end{array}$ & $\begin{array}{r}60.5 \\
51.6-66.0\end{array}$ \\
\hline $\begin{array}{l}\text { CD4 Cells (count } / \mathrm{mm} 3 \\
\text { Mean } \\
\text { Range }\end{array}$ & $\begin{array}{r}513 \\
248-776\end{array}$ & $\begin{array}{r}489 \\
396-575\end{array}$ & $\begin{array}{r}572 \\
305-1,065\end{array}$ \\
\hline $\begin{array}{l}\text { Viral load (copies/ml) } \\
\text { Mean } \\
\text { Range }\end{array}$ & $\begin{array}{r}1.8 \times 10^{5} \\
1.96 \times 10^{3} \\
8.08 \times 10^{5} \\
\end{array}$ & $\begin{array}{l}3.36 \times 10^{5} \\
2.20 \times 10^{4} \\
1.19 \times 10^{6}\end{array}$ & $\begin{array}{r}6.29 \times 10^{4} \\
<400- \\
1.43 \times 10^{5} \\
\end{array}$ \\
\hline $\begin{array}{l}\text { Cholesterol }(\mathrm{mmol} / \mathrm{L}) \\
\text { Normal value }-5.18-6.19 \\
\text { Mean } \\
\text { Range }\end{array}$ & $\begin{array}{r}3.95 \\
3.35-4.59\end{array}$ & $\begin{array}{r}4.61 \\
4.15-5.46\end{array}$ & $\begin{array}{r}4.61 \\
3.63-5.78\end{array}$ \\
\hline $\begin{array}{l}\text { Triglyceride }(\mathrm{mmol} / \mathrm{L}) \\
\text { Normal value } 0.45-1.69 \\
\text { Mean } \\
\text { Range }\end{array}$ & $\begin{array}{r}1.48 \\
0.6-2.31\end{array}$ & $\begin{array}{r}1.36 \\
0.6-2.7\end{array}$ & $\begin{array}{r}1.38 \\
0.3-3.2\end{array}$ \\
\hline $\begin{array}{l}\text { HDL }(\mathrm{mmol} / \mathrm{L}) \\
\text { Normal value }<0.91 \\
\text { Mean } \\
\text { Range }\end{array}$ & $\begin{array}{r}0.74 \\
0.57-1.17\end{array}$ & $\begin{array}{r}0.65 \\
0.57-0.72\end{array}$ & $\begin{array}{r}0.70 \\
0.56-1.10\end{array}$ \\
\hline $\begin{array}{l}\text { LDL (mmol/L } \\
\text { Normal value } 3.36-4.11 \\
\text { Mean } \\
\quad \text { Range }\end{array}$ & $\begin{array}{r}2.62 \\
2.09-3.3\end{array}$ & $\begin{array}{r}2.92 \\
2.09-3.81\end{array}$ & $\begin{array}{r}3.55 \\
2.05-4.95\end{array}$ \\
\hline $\begin{array}{l}\text { ALT }(\mathrm{U} / \mathrm{L}) \\
\text { Normal value } 30-115 \\
\quad \text { Mean } \\
\quad \text { Range }\end{array}$ & $\begin{array}{r}34.0 \\
20-70\end{array}$ & $\begin{array}{r}28.6 \\
14-45\end{array}$ & $\begin{array}{r}125.0 \\
20-460\end{array}$ \\
\hline $\begin{array}{l}\text { AST }(\mathrm{U} / \mathrm{L}) \\
\text { Normal value 9-40 } \\
\text { Mean } \\
\text { Range }\end{array}$ & $\begin{array}{r}28.2 \\
11-65\end{array}$ & $\begin{array}{l}22.0 \\
8-39\end{array}$ & $\begin{array}{r}118.0 \\
12-450\end{array}$ \\
\hline $\begin{array}{l}\text { BUN }(\mathrm{mmol} / \mathrm{L}) \\
\text { Normal value } 2.8-8.9 \\
\text { Mean } \\
\text { Range }\end{array}$ & $\begin{array}{r}3.67 \\
3.21-3.96\end{array}$ & $\begin{array}{r}3.64 \\
3.45-3.80\end{array}$ & $\begin{array}{r}3.52 \\
3.25-3.85\end{array}$ \\
\hline $\begin{array}{l}\text { Creatinine } \quad(\mu \mathrm{molL}) \\
\text { Normal value } 53-133 \\
\quad \text { Mean } \\
\quad \text { Range }\end{array}$ & $\begin{array}{r}68.2 \\
53-75\end{array}$ & $\begin{array}{r}64 \\
56-74\end{array}$ & $\begin{array}{r}58.2 \\
52-67\end{array}$ \\
\hline $\begin{array}{l}\text { Hemoglobin }(\mathrm{g} / \mathrm{L}) \\
\text { Normal value } 12-16 \\
\text { Mean } \\
\text { Range }\end{array}$ & $\begin{array}{r}14.4 \\
10.1-19.7 \\
\end{array}$ & $\begin{array}{r}12.96 \\
11.9-14.8 \\
\end{array}$ & $\begin{array}{r}14.32 \\
12.4-16.4 \\
\end{array}$ \\
\hline
\end{tabular}


Table 2. Change in viral load from baseline to 3 months after treatment among 3 treatment groups. Monolaurin clinical trial

\begin{tabular}{|l|r|r|r|r|}
\hline Change in viral load & Coconut oil & $\begin{array}{c}\text { Low dose } \\
\text { monolaurin }\end{array}$ & $\begin{array}{c}\text { High dose } \\
\text { monolaurin }\end{array}$ & \multicolumn{1}{c|}{ Total } \\
\hline Decrease -no./\% & $\mathbf{3 ( 6 0 )}$ & $\mathbf{2 ( 4 0 )}$ & $\mathbf{1}(\mathbf{2 0})$ & $\mathbf{6 ( 4 0 . 0 )}$ \\
No change no./\% & $0(0)$ & $0(0)$ & $1(20)$ & $1(6.7)$ \\
Increase - no./\% & $2(40)$ & $3(60)$ & $3(60)$ & $8(53.3)$ \\
Total & $5(100)$ & $5(100)$ & $5(100)$ & $15(100)$ \\
\hline
\end{tabular}

Table 3. Change in viral load from baseline to 6 months after treatment among 3 treatment groups. Monolaurin clinical trial

\begin{tabular}{|l|r|r|r|r|}
\hline Change in viral load & Coconut oil & $\begin{array}{c}\text { Low dose } \\
\text { monolaurin }\end{array}$ & $\begin{array}{c}\text { High dose } \\
\text { monolaurin }\end{array}$ & \multicolumn{1}{c|}{ Total } \\
\hline Decrease -no./\% & $\mathbf{3 ( 6 0 )}$ & $\mathbf{4 ( 8 0 )}$ & $\mathbf{3 ( 6 0 )}$ & $\mathbf{1 0}(\mathbf{6 6 . 7 )}$ \\
No change no./\% & $2(40)$ & $0(0)$ & $1(20)$ & $3(20)$ \\
Increase - no./\% & $0(0)$ & $1(20)$ & $1(20)$ & $1(13.3)$ \\
Total & $5(100)$ & $5(100)$ & $5(100)$ & $15(100)$ \\
\hline
\end{tabular}

Table 4. Change in CD4 count from baseline to 3 months after treatment among 3 treatment groups. Monolaurin clinical trial

\begin{tabular}{|l|r|r|r|r|}
\hline \multicolumn{1}{|c|}{ Change in CD4 } & Coconut oil & $\begin{array}{c}\text { Low dose } \\
\text { monolaurin }\end{array}$ & $\begin{array}{c}\text { High dose } \\
\text { monolaurin }\end{array}$ & \multicolumn{1}{c|}{ Total } \\
\hline Increase $-\mathbf{n o .} / \%$ & $\mathbf{1}(\mathbf{2 0})$ & $\mathbf{0 ( 0 )}$ & $\mathbf{2 ( 4 0 )}$ & $\mathbf{3 ( 2 0 . 0 )}$ \\
Decrease - -no./\% & $4(80)$ & $5(100)$ & $3(60)$ & $12(80.0)$ \\
Total & $5(100)$ & $5(100)$ & $5(100)$ & $15(100)$ \\
\hline
\end{tabular}

Table 5. Change in CD4 count from baseline to 6 months after treatment among 3 treatment groups. Monolaurin clinical trial

\begin{tabular}{|l|r|r|r|r|}
\hline \multicolumn{1}{|c|}{ Change in CD4 } & Coconut oil & $\begin{array}{r}\text { Low dose } \\
\text { monolaurin }\end{array}$ & $\begin{array}{r}\text { High dose } \\
\text { monolaurin }\end{array}$ & Total \\
\hline Increase- no./\% & $\mathbf{3 ( 6 0 )}$ & $\mathbf{1 ( 2 0 )}$ & $\mathbf{3}(\mathbf{6 0})$ & $\mathbf{7 ( 4 6 . 7 )}$ \\
Decrease - no./\% & $2(40)$ & $4(80)$ & $2(40)$ & $8(53.3)$ \\
Total & $5(100)$ & $5(100)$ & $5(100)$ & $15(100$ \\
\hline
\end{tabular}


Table 6. Total log-change in viral load 3 months and 6 months after treatment among 3 treatment groups. Monolaurin clinical trial

\begin{tabular}{|l|l|l|l|}
\hline \multicolumn{1}{|c|}{$\begin{array}{c}\text { Months after } \\
\text { treatment }\end{array}$} & \multicolumn{1}{|c|}{ Coconut oil } & Low dose monolaurin & \multicolumn{1}{c|}{$\begin{array}{c}\text { High dose } \\
\text { monolaurin }\end{array}$} \\
\hline 3 months & +0.095003622 & +0.608952472 & +0.013335636 \\
6 months & -0.055844178 & -0.846177528 & -0.48687464 \\
\hline
\end{tabular}

Table 7. Total change in CD4 count 3 months \& 6 months after treatment among 3 treatment groups. Monolaurin clinical trial

\begin{tabular}{|l|c|c|c|}
\hline \multicolumn{1}{|c|}{$\begin{array}{c}\text { Months after } \\
\text { treatment }\end{array}$} & Coconut oil t oil & $\begin{array}{c}\text { Low dose } \\
\text { monolaurin }\end{array}$ & $\begin{array}{c}\text { High dose } \\
\text { monolaurin }\end{array}$ \\
\hline 3 months & -595 & -1050 & -719 \\
6 months & +221 & -533 & +89 \\
\hline
\end{tabular}

Table 8 Blood chemistries 6 months after treatment among 3 treatment groups. Monolaurin clinical trial

\begin{tabular}{|c|c|c|c|}
\hline $\begin{array}{c}6 \text { months after } \\
\text { treatment }\end{array}$ & Coconut oil & $\begin{array}{l}\text { Low dose } \\
\text { monolaurin }\end{array}$ & $\begin{array}{c}\text { High dose } \\
\text { monolaurin }\end{array}$ \\
\hline $\begin{array}{r}\text { Cholesterol } \\
\text { Mean } \\
\text { Range }\end{array}$ & $\begin{array}{r}5.08 \\
4.57-5.71\end{array}$ & $\begin{array}{r}5.17 \\
4.0-5.95\end{array}$ & $\begin{array}{r}5.41 \\
4.3-6.09\end{array}$ \\
\hline $\begin{array}{r}\text { Triglyceride } \\
\text { Mean } \\
\text { Range } \\
\end{array}$ & $\begin{array}{r}2.53 \\
0.66-6.0 \\
\end{array}$ & $\begin{array}{r}1.84 \\
1.2-2.8 \\
\end{array}$ & $\begin{array}{r}2.42 \\
1.0-4.6 \\
\end{array}$ \\
\hline $\begin{array}{l}\text { HDL } \\
\quad \text { Mean } \\
\text { Range }\end{array}$ & $\begin{array}{r}0.75 \\
0.36-1.25 \\
\end{array}$ & $\begin{array}{r}0.58 \\
0.24-0.74 \\
\end{array}$ & $\begin{array}{r}1.52 \\
0.62-4.6\end{array}$ \\
\hline $\begin{array}{ll}\text { LDL } & \\
& \text { Mean } \\
& \text { Range } \\
\end{array}$ & $\begin{array}{r}3.56 \\
3.08-4.18 \\
\end{array}$ & $\begin{array}{r}3.0 \\
0.49-3.82 \\
\end{array}$ & $\begin{array}{r}3.51 \\
2.04-4.51 \\
\end{array}$ \\
\hline $\begin{array}{ll}\text { ALT } & \\
& \text { Mean } \\
& \text { Range }\end{array}$ & $\begin{array}{r}55.8 \\
20-92\end{array}$ & $\begin{array}{r}96 \\
30-173\end{array}$ & $\begin{array}{r}124.8 \\
20-464\end{array}$ \\
\hline $\begin{array}{ll}\text { AST } & \\
& \text { Mean } \\
& \text { Range }\end{array}$ & $\begin{array}{r}49.6 \\
11-86\end{array}$ & $\begin{array}{r}88.2 \\
22-166\end{array}$ & $\begin{array}{r}117.4 \\
14-455\end{array}$ \\
\hline $\begin{array}{ll}\text { BUN } & \\
& \text { Mean } \\
& \text { Range }\end{array}$ & $\begin{array}{r}3.88 \\
2.6-5.0\end{array}$ & $\begin{array}{r}3.37 \\
2.65-3.7\end{array}$ & $\begin{array}{r}3.57 \\
2.5-4.94\end{array}$ \\
\hline $\begin{array}{r}\text { Creatinine } \\
\text { Mean } \\
\text { Range }\end{array}$ & $\begin{array}{r}69.6 \\
42-101\end{array}$ & $\begin{array}{r}53.6 \\
44-62\end{array}$ & $\begin{array}{r}58 \\
43-86\end{array}$ \\
\hline $\begin{array}{r}\text { Hemoglobin } \\
\text { Mean } \\
\text { Range }\end{array}$ & $\begin{array}{r}12.9 \\
9.2-15.4\end{array}$ & $\begin{array}{r}12.1 \\
9.9-15.5\end{array}$ & $\begin{array}{r}14.34 \\
12.2-16.6\end{array}$ \\
\hline
\end{tabular}


Table 9. Mean change in patients' weight (kgs) 3 months and 6 months after treatment among 3 treatment groups. Monolaurin clinical trial

\begin{tabular}{|l|c|c|c|}
\hline $\begin{array}{c}\text { Months after } \\
\text { treatment }\end{array}$ & Coconut oil & $\begin{array}{c}\text { Low dose } \\
\text { monolaurin }\end{array}$ & $\begin{array}{c}\text { High dose } \\
\text { monolaurin }\end{array}$ \\
\hline 3 months & 4.0 & 6.2 & 0.5 \\
6 months & 1.7 & 3.1 & 1.5 \\
\hline
\end{tabular}

Table 10. Adverse reactions, all treatment groups. Monolaurin Clinical Trial

\begin{tabular}{|l|l|l|l|}
\hline $\begin{array}{c}\text { Months after } \\
\text { treatment }\end{array}$ & \multicolumn{1}{|c|}{ Oil } & \multicolumn{1}{|c|}{ Low } & \multicolumn{1}{c|}{ High } \\
\hline 3 moths & $\bullet$ Acne & $\bullet$ acne & $\bullet$ feeling of warmth \\
& $\bullet$ feeling of warmth & $\bullet$ green urine & $\bullet$ green urine \\
& $\bullet$ enlarged lymph nodes & $\bullet$ enlarged lymph nodes & $\bullet$ abdominal fullness \\
& $\bullet$ furuncle & $\bullet$ sebaceous cyst & $\bullet$ enlarged lymph nodes \\
& $\bullet$ abdominal fullness & & $\bullet$ irregular bowel movement \\
& $\bullet$ headache & & $\bullet$ headache \\
\hline 6 months & $\bullet$ acne & $\bullet$ papules & $\bullet$ carbuncle \\
& $\bullet$ fever, ascites, dysuria & $\bullet$ sebaceous cyst & $\bullet$ enlarged lymph nodes \\
& & $\bullet$ enlarged nymph nodes & $\bullet$ tonsillitis \\
& & $\bullet$ tonsillitis & $\bullet$ fullness \\
\hline
\end{tabular}



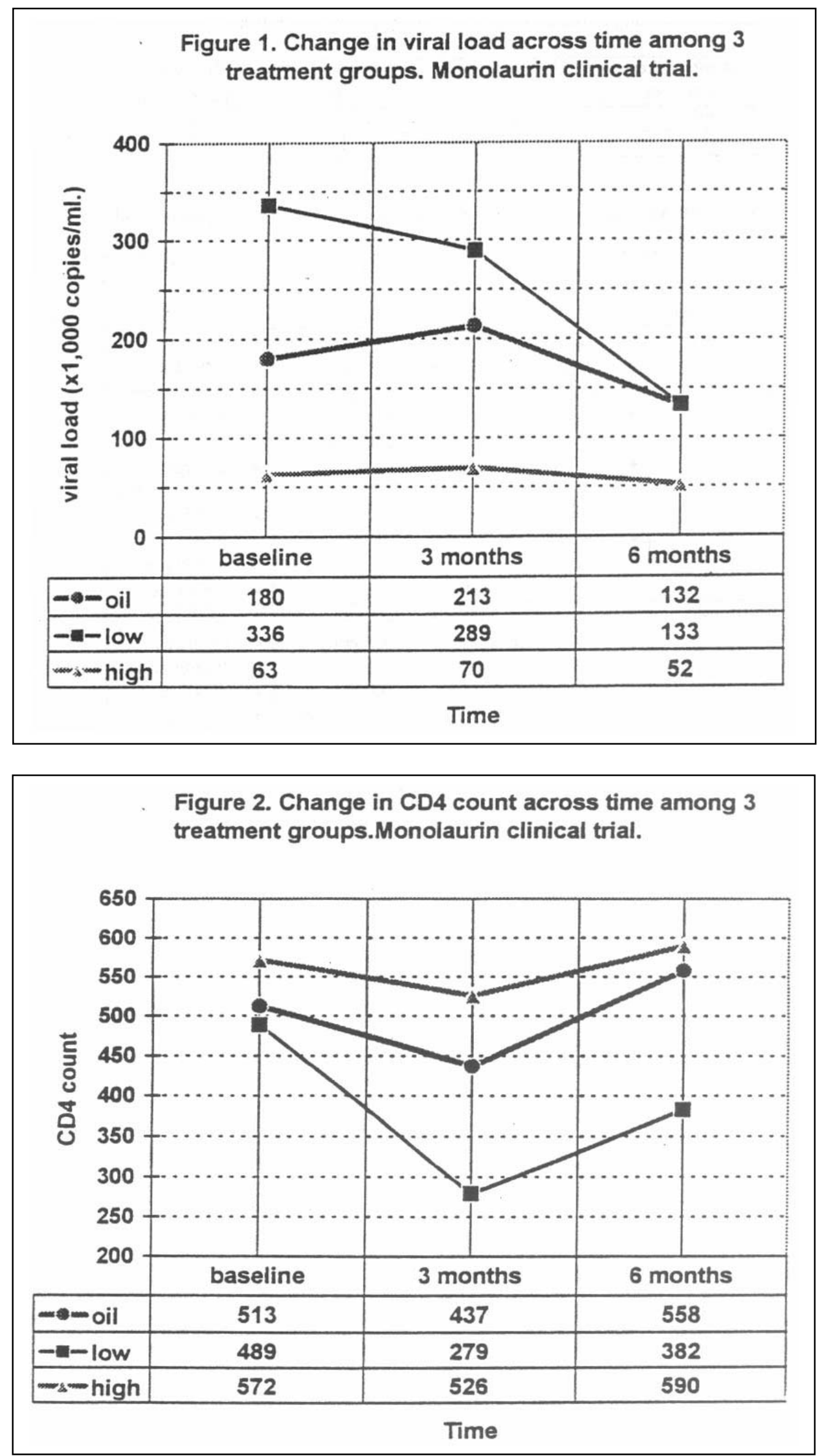